\title{
Low-level inflammation promotes bone growth
}

Ectopic bone formation and spinal fusion are important contributors to disease severity in ankylosing spondylitis (AS). Previous studies have linked inflammation to new bone growth in AS, but the exact pathways involved were unknown. Now, new evidence highlights a role for Wnt proteins in bridging the gap between inflammation and bone formation.

"Two main theories exist regarding the relationship between inflammation and ectopic new bone formation in AS," explains corresponding author Hui Liu. "One theory postulates that inflammation triggered by unknown stimuli drives a bone catabolic process and is followed by overreactive osteogenesis. The other theory suggests that inflammation and bone formation are uncoupled and that the same triggers independently activate inflammatory and stromal cells."

To investigate the potential mechanisms behind these theories, Liu and colleagues developed an in vitro cell culture system that mimicked the cellular microenvironment at sites of ectopic bone formation. This system enabled the researchers to mix and match bone progenitor cells from different sources with varying levels of TNF to establish whether the intensity of inflammation has a role in bone formation.

Conditioned media from monocytes consistently stimulated with low-dose TNF induced the expression of several Wnt proteins and prompted new bone formation, whereas conditioned media from monocytes stimulated with highdose TNF induced expression of the Wnt agonist Dickkopf-related protein 1, a suppressor of bone formation. Using gene knockdown experiments in the same in vitro system, the researchers also showed that both canonical and non-canonical Wnt signalling pathways were required for new bone formation.

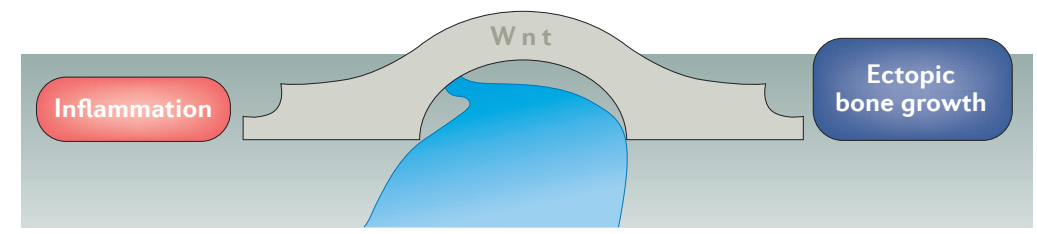

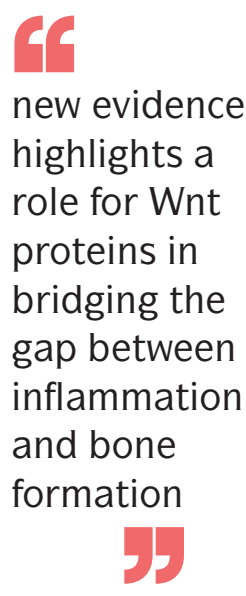

new evidence

highlights a

role for Wnt

proteins in

bridging the

gap between

inflammation

and bone

formation

Macmillan Publishers Limited

The association between the expression of Wnt proteins and new bone formation was reinforced by the results from two mouse models of AS, a modified collagen-induced arthritis model and a proteoglycaninduced spondylitis model. In these mice, blocking either canonical or non-canonical Wnt signalling pathways reduced ectopic bone formation, an effect that was greatly enhanced by blocking both pathways simultaneously.

A link between bone formation and the level of inflammation was not explicitly shown in these in vivo experiments, but previous studies in patients with AS have shown an association between suppression of inflammation and an increase in ectopic bone formation.

"The osteogenic molecules induced by pro-inflammatory cytokines represent a key link between inflammation and new bone formation, in which inflammation intensity acts as a bone formation switch," concludes Liu.

Joanna Collison

ORIGINAL ARTICLE Li, X. et al. Inflammation intensity-dependent expression of osteoinductive Wnt proteins is critical for ectopic new bone formation in ankylosing spondylitis. Arthritis Rheumatol. https://doi.org/10.1002/art.40468 (2018) 


\section{ONLINE ONLY}

\section{Subject categories:}

Health sciences / Diseases / Rheumatic diseases / Spondyloarthritis /

Ankylosing spondylitis

[URI /692/699/1670/2766/1827]

Health sciences / Medical research / Experimental models of disease [URI /692/308/1426]

Biological sciences / Developmental biology / Bone remodelling [URI /631/136/815] 